\title{
O papel das MMP-2 e -9 no desenvolvimento do carcinoma epidermóide
}

\author{
The role of MMP-2 and MMP-9 in the development of squamous cell carcinoma
}

\author{
Andresa Costa Pereira', Elaine Dias do Carmo', Vanessa Ávila Sarmento Silveira', Susana Ungaro Amadei', Luiz Eduardo Blumer Rosa²
}

\section{Resumo}

No carcinoma epidermóide, os processos de invasão e metástase são regulados por um complexo sistema dependente da interação entre as células neoplásicas e as células estromais hospedeiras. Esses processos ocorrem como conseqüência à degradação da membrana basal e da matriz extracelular por diversas enzimas, dentre as quais as metaloproteinases da matriz (MMP) apresentam destaque. Essas são enzimas zinco-dependentes, pouco expressas em tecidos normais e substancialmente aumentadas na maioria das neoplasias malignas. Dentre todas as MMP conhecidas, as MMP-2 e -9 (gelatinases) têm sido consistentemente associadas com a agressividade, o potencial metastático e o prognóstico desfavorável das neoplasias malignas. As gelatinases desempenham um papel fundamental no processo da carcinogênese, pois degradam principalmente colágeno tipo IV, componente fundamental da membrana basal, participando do processo de invasão do estroma e invasão dos vasos sangüíneos, processo fundamental para a metástase. Portanto, o objetivo desse trabalho foi apresentar uma revisão dos aspectos gerais das MMP, abordando principalmente as MMP-2 e -9, bem como suas relaçôes com o desenvolvimento do carcinoma epidermóide, buscando as possibilidades de prevenção e tratamento.

Palavras-Chave: Carcinoma de células escamosas, Neoplasias, Metástase neoplásica, Gelatinase A, Gelatinase B.

\section{Abstract}

In squamous cell carcinoma, invasion and metastasis are regulated by a complex system characterized by neoplastic and stromal cell interaction. These processes are a consequence of basement membrane and extracellular matrix degradation by various enzymes, mainly matrix metalloproteinases (MMPs). The latter are zinc-dependent enzymes, with low expression in normal tissues but over-expressed in malignant neoplasms. Of all MMPs, MMP-2 and MMP-9 (gelatinases) have been consistently associated with aggressiveness, metastatic potential, and poor prognosis in malignant neoplasms. Gelatinases show a fundamental function in carcinogenesis, since they degrade type IV collagen, an important basement membrane component, participating in stromal and vascular invasion, a fundamental process related to metastasis. This paper aimed to review general aspects of MMPs, especially MMP-2 and MMP9 , and their relationship to the development of squamous cell carcinoma, with a view towards their preventive and therapeutic possibilities.

Key words: Squamous cell carcinoma, Cancer, Metastasis, MMP-2, MMP-9.

\footnotetext{
Trabalho realizado no Departamento de Biociências e Diagnóstico Bucal, Disciplina de Patologia Bucal - Faculdade de Odontologia de São José dos Campos, UNESP.

${ }^{1}$ Doutorandas em Biopatologia Bucal - Faculdade de Odontologia de São José dos Campos, UNESP.

${ }^{2}$ Professor Doutor da Disciplina de Patologia Bucal - Faculdade de Odontologia de São José dos Campos, UNESP.

Endereço para correspondência: Andresa Costa Pereira - Av. Engenheiro Francisco José Longo, 777 - Jardim São Dimas - Cep: 12.245-000 - São José dos Campos - SP - Brasil. E-mail: andresa-cp@uol.com.br.
} 


\section{INTRODUÇÃO}

Segundo estimativas do Instituto Nacional de Câncer (INCA), em 2005, serão contabilizados 467.440 novos casos de câncer no Brasil, sendo 229.610 no sexo masculino e 237.830 no feminino ${ }^{1}$. Esses valores são extremamente elevados e impulsionam numerosos estudos que visam ao entendimento do processo de carcinogênese, bem como a busca de mecanismos preventivos ou tratamentos mais efetivos para o câncer.

As células neoplásicas originam-se de células normais que sofreram a ação de um ou mais agentes cancerígenos, os quais provocaram alteraçōes permanentes no DNA. A partir dessa agressão, as células que estão no ciclo celular, ou que são capazes de nele entrar darão origem a clones de células transformadas ${ }^{2}$.

O carcinoma epidermóide ou espino-celular (CEC) é uma neoplasia maligna de células do tecido epitelial ${ }^{3,4,5}$. A determinação da capacidade de invasão e metástase de um CEC é extremamente importante na conduta clínica, no tratamento e no prognóstico ${ }^{6}$.

Um acontecimento fundamental para o processo de invasão é a migração das células epiteliais neoplásicas rompendo o limite com o tecido conjuntivo. Para que esse fenômeno ocorra, são necessárias alterações nas interações entre as células neoplásicas e a matriz extracelular (MEC) e aumento na secreção das enzimas degradadoras da $\mathrm{MEC}^{6,7,8}$. Longe de ser uma barreira física, o estroma participa ativamente do processo de carcinogênese $e^{7,89}$.

Dentre as enzimas degradadoras da MEC, destacamse as metaloproteinases da matriz (MMP) ${ }^{8,10,11,12}$. Essas podem agir em um ou mais componentes ${ }^{13}$, sendo que juntas têm a capacidade de degradar todos os elementos protéicos conhecidos da $\mathrm{MEC}^{5}$.

\section{Metaloproteinases da MATRIZ EXTRACELULAR}

As MMP pertencem a uma família de enzimas que necessitam da presença do zinco para realizarem suas funçôes ${ }^{13,14}$. São classificadas de acordo com seu domínio e organização estrutural, além do substrato específico para degradaçãa ${ }^{11}$. Apresentam diversas classes, dentre elas, as colagenases, as gelatinases, as estromelisinas, as matrilisinas e as MMP ligadas à membrana (MTMMP) $)^{11,13,15}$.

As colagenases 1, 2 e 3 são as MMP-1, -8 e -13, respectivamente. Essas degradam as moléculas de colágeno intersticial tipos I, II e III, além de digerirem outras moléculas da MEC 5,16 .

As gelatinases A e B (MMP-2 e -9) degradam principalmente colágeno desnaturado (gelatinas) e colágeno tipo $\mathrm{IV}^{5,13}$, enquanto as estromelisinas 1 e 2 (MMP-3 e -10) podem degradar fibronectina e proteoglicanas $^{16,17}$.

As matrilisinas 1 e 2 (MMP-7 e -26, respectivamente) digerem diversos componentes da matriz, dentre os quais estão a fibronectina e o colágeno tipo $\mathrm{IV}^{5,16}$.

As MT-MMP são numeradas de 1 a 6: MT1-MMP, MT2-MMP, MT3-MMP, MT4-MMP, MT5-MMP e MT6-MMP, correspondendo respectivamente às MMP$14,-15,-16,-17,-24$ e -25. Além de apresentarem papel importante na ativação das outras $\mathrm{MMP}^{18}$, os subtipos das MT-MMP são responsáveis pela degradação de diferentes componentes da MEC ${ }^{5,17}$. A nomenclatura e os substratos específicos de cada grupo das MMP estão resumidos na tabela 1 .

Em condições fisiológicas normais, há uma rigorosa regulação da secreção das MMP, as quais são sintetizadas e secretadas como pró-enzimas, zimógenos, que posteriormente serão ativadas $s^{13,15,19,20}$. Essa regulação ocorre apenas em momentos específicos, nos quais existem processos multifásicos de ativação dos zimógenos, além de haver vários inibidores sangüíneos e teciduais para monitorar a ação da proteinase ${ }^{13}$. O controle da quantidade de enzima ativa é feito pelos inibidores teciduais das MMP (TIMP) ${ }^{13,21,22}$. O desequilíbrio entre MMP e TIMP pode resultar em várias patologias ${ }^{22}$.

Os níveis das MMP nos tecidos saudáveis são baixos ou praticamente indetectáveis ${ }^{15,23}$, entretanto, sua expressão é substancialmente aumentada na maioria das neoplasias malignas, apresentando importante ação proteolítica nos processos de invasão e metástase $^{5,8,12,15,24,25,26}$. Neste aspecto, as MMP-2 e -9 têm um papel crucial na progressão tumoral, atuando diretamente sobre os componentes da membrana basal (MB). Atualmente, é aceito que a MB, a qual separa o epitélio do tecido conjuntivo, representa a primeira estrutura a ser degradada durante o processo de invasão das células epiteliais neoplásicas ${ }^{8,27}$, fato pelo qual as gelatinases mereceram destaque principal nesta revisão.

\section{Processos de inVASÃo e metástase}

As células do CEC atravessam a MB e penetram na matriz extracelular, objetivando invadir estruturas adjacentes, fenômeno que caracteriza o processo de invasão neoplásica ${ }^{28}$.

Uma das mais sérias conseqüências da invasão é a produção de metástases. As células malignas, da mesma forma que foram capazes de romper a $\mathrm{MB}$ e degradar o 
Tabela 1. Nomenclatura e alguns substratos específicos dos principais grupos das metaloproteinases da matriz

\begin{tabular}{lll}
\hline \multicolumn{1}{c}{ Proteína } & MMP & \multicolumn{1}{c}{ Alguns substratos específicos } \\
\hline $\begin{array}{c}\text { Colagenases } \\
\text { Colagenase 1 }\end{array}$ & Colágenos I, II e III \\
Colagenase 2 & MMP-1 & \\
Colagenase 3 & MMP-13 & \\
Gelatinases & & Gelatina e colágeno IV \\
Gelatinase A & MMP-2 & \\
Gelatinase B & MMP-9 & \\
Estromelisinas & & Fibronectina e proteoglicanas \\
Estromelisina 1 & MMP-3 & \\
Estromelisina 2 & MMP-10 & \\
Matrilisinas & & Fibronectina e colágeno IV \\
Matrilisina 1 & MMP-7 & \\
Matrilisina 2 & MMP-26 & \\
MMP ligadas à membrana & & \\
MT1-MMP & $M M P-14$ & Colágenos I, II e III, gelatina e fibronectina \\
MT2-MMP & $M M P-15$ & Fibronectina e laminina \\
MT3-MMP & $M M P-16$ & Colágeno III e fibronectina \\
MT4-MMP & $M M P-17$ & Gelatina e fibronectina \\
MT5-MMP & MMP-24 & Proteoglicanas \\
MT6-MMP & MMP-25 & Gelatina \\
\hline
\end{tabular}

MMP: metaloproteinase da matriz, MT-MMP: MMP ligadas à membrana

tecido intersticial, podem invadir os vasos sangüíneos e linfáticos e serem levadas pela circulação ${ }^{3}$. Estando na corrente sangüínea, necessitam ainda atravessar as células endoteliais e a $\mathrm{MB}$ em um local distante, e só então emigrarem do vaso para um tecido íntegro, no qual irão se proliferar e caracterizar o processo de metástase ${ }^{6}$.

Para a degradação da $\mathrm{MB}$, é necessária a presença de enzimas proteolíticas ${ }^{6,10}$, dentre as quais as gelatinases apresentam grande importância. Como principais funçōes, as MMP-2 e -9 fragmentam laminina e colágeno tipo IV, componentes fundamentais da $\mathrm{MB}^{8,14,17}$.

Devido ao papel importante das gelatinases no desenvolvimento do câncer, diversos autores têm relacionado os níveis de MMP-2 e -9 com carcinomas de esôfago ${ }^{29}$, oral ${ }^{30,31}$, de endométrio ${ }^{32}$, de pele $^{28}$, de laringe ${ }^{33}$, entre outros.

Os estudos para detecção da influência dos níveis de MMP no desenvolvimento do câncer são baseados em avaliações provenientes de biópsias humanas ou de pesquisas pré-clínicas in vivo e in vitro ${ }^{15}$.

Kawamata et al. ${ }^{34}$ verificaram que a indução da expressão de MMP-2 em uma linhagem de células de carcinoma de bexiga de rato aumentou a capacidade de metástase deste modelo. Comparando sistemas metastáticos e não-metastáticos, Bernhard et al. ${ }^{35}$ verificaram que a transfecção de MMP-9 conferiu capacidade de metástase às células não-metastáticas. Em adição, em modelo de sarcoma de rato, Hua $\&$ Muschel $^{36}$ verificaram que a inibição da expressão da MMP-9 neste modelo reduziu a capacidade de metástase. Desta maneira, ambos os estudos ${ }^{35,36}$ relacionaram o aumento dos níveis de MMP-9 com a elevação do potencial de metástase, enquanto que Kawamata et al..$^{34}$ demonstraram a mesma relação com respeito à MMP-2.

A maioria dos estudos para detecção dos níveis das MMP-2 e -9 utiliza técnicas de imunoistoquímica ${ }^{6,28}$ e zimografia ${ }^{29,37}$. O estudo imunoistoquímico com anticorpos mono ou policlonais contra MMP-2 e -9 pode apresentar a localização de cada MMP no tecido tumoral e pode ser comparado com os achados em cortes histopatológicos convencionais, entretanto, pode, às vezes, resultar em falso positivo baseado em reações cruzadas não específicas ${ }^{38}$. Já a zimografia demonstra a ação proteolítica mais do que a antigenicidade e assim fornece medidas semiquantitativas das MMP no tumor, além de distinguir a MMP de forma ativa da proenzima $^{37}$. Desta maneira, a avaliação concomitante da zimografia e da imunoistoquímica mostra-se 
importante na detecção das MMP.

$\mathrm{Na}$ literatura, é relatado que altos níveis das MMP2 e -9 estão presentes em casos com grande potencial de invasão ${ }^{29,37}$ e metástase ${ }^{6,28}$. Koyama et al.$^{29}$ avaliaram, por imunoistoquímica e zimografia, 30 casos de CEC de esôfago e demonstraram que tanto a MMP-2 quanto a MMP-9 estavam relacionadas ao processo de invasão tumoral. Concordando com estes achados, Ikebe et al. ${ }^{37}$ estudaram 57 amostras de tumores epidermóides de cabeça e pescoço e, da mesma maneira, demonstraram que os níveis imunoistoquímicos e zimográficos de MMP-2 e -9 estavam correlacionados com o potencial de invasão. Hong et al. ${ }^{6}$ avaliaram 19 casos metastáticos de CEC oral e 25 não-metastáticos, também por imunoistoquímica e zimografia, e verificaram que os níveis de MMP-2 e -9 são indicadores do potencial de invasão, sendo que a freqüência da expressão da MMP9 foi maior do que da MMP-2. Fundyler et al. ${ }^{28}$, utilizando apenas marcação imunoistoquímica para MMP-2, demonstraram a relação entre esta enzima e a capacidade de invasão e metástase.

No entanto, a relação entre a expressão das MMP e a capacidade de metástases do CEC ainda apresenta-se controversa ${ }^{37}$. Hong et al. ${ }^{6}$ relataram que os níveis de MMP-2 e -9 são indicadores do potencial metastático e, em particular a MMP-9 pode ser um bom marcador como fator prognóstico. Entretanto, o estudo de Ikebe et $\mathrm{al}^{37}$ demonstrou que apesar dos níveis de MMP-2 e -9 terem sido associados ao potencial de invasão, estes não foram relacionados com o potencial de metástase, questionando o papel ainda não completamente claro das MMP como fator prognóstico.

Apesar de serem necessários mais estudos, avaliando a relação entre a expressão das MMP e a capacidade de metástase, vale salientar que há uma concordância entre os autores no que diz respeito à importância destas enzimas na capacidade de invasão das neoplasias. Notada a relevância deste fato, deve-se destacar que a detecção precoce dos níveis aumentados das gelatinases pode apresentar-se importante na realização de um correto diagnóstico e um tratamento eficaz.

A associação das técnicas de imunoistoquímica e zimografia pode ser utilizada mais rotineiramente, visto que estas promovem um resultado confiável, além de serem métodos de fácil execução e custo acessível. Podese sugerir o uso dessas técnicas para a elaboração de um protocolo de rotina que forneça dados dos níveis das gelatinases das lesões enviados para os laboratórios de patologia cirúrgica. Como exemplo, a avaliação dos níveis das MMP-2 e -9 em lesões pré-malignas, como biópsias incisionais de leucoplasias extensas e inoperáveis, poderia auxiliar a detecção de locais com maior predisposição à transformação maligna e invasão. Este procedimento poderia servir como fator prognóstico no curso clínico do CEC, facilitando a conduta do cirurgião.

As MMP também têm sido foco de atenção, objetivando o uso de fármacos ou fatores que possam regular a expressão dessas enzimas ${ }^{14}$. Diversos fármacos inibidores das MMP têm sido sintetizados para estudos no tratamento de neoplasias malignas ${ }^{24,25,26,39,40}$. Dentre estes medicamentos, podemos citar os peptideomiméticos, os não-peptideomiméticos, os derivados da tetraciclina e os bisfosfonatos ${ }^{15}$, sendo que todos interferem na expressão e ativação das MMP, reduzindo a função dessas enzimas e conseqüente degradação da MEC. Dessa maneira, tem-se buscado a inibição da função das MMP como uma forma alternativa de terapia anti-câncer ${ }^{15,41,42}$.

Devido ao fato de existirem diversos tipos de inibidores sintéticos das MMP, ainda há algumas controvérsias quanto aos resultados obtidos com o uso destes fármacos. Santos et al. ${ }^{39}$ avaliaram a ação de diversos compostos inibidores das MMP (AG3287, AG3293, AG3294, AG3296, AG3319 e AG3340), utilizando um modelo de carcinoma pulmonar. Esses autores verificaram que o AG3340 foi o mais eficaz na inibição do crescimento neoplásico, entretanto, outros compostos, como o AG3293 e o AG3294, não apresentaram ação anti-tumoral. Já Wylie et al. ${ }^{24}$ verificaram que o batimastat (BB-94), um outro inibidor sintético da MMP, reduziu o tamanho, mas não o número de metástases de melanoma no fígado de camundongos. Avaliando a ação do MMI-166, um inibidor com espectro seletivo para gelatinases, Maekawa et al. ${ }^{25}$ verificaram, em vários modelos de metástases, que esse fármaco reduziu significativamente o crescimento de metástase tumoral em pulmão, fígado e cavidade peritoneal, in vivo, apesar de não afetar $o$ crescimento neoplásico de células in vitro.

Pode-se verificar que, embora a literatura mostre que alguns fármacos não obtiveram um completo sucesso, o desenvolvimento de um novo inibidor das MMP que seja menos tóxico e mais efetivo tem sido foco de diversos estudos ${ }^{15,24,25,39}$.

\section{CONCLUSÃO}

Diversos estudos têm se focado nas MMP, devido ao seu papel importante no desenvolvimento do CEC. As MMP-2 e -9 merecem destaque por participarem 
principalmente dos processos de invasão e metástase. Essas enzimas têm sido estudadas na busca da detecção imunoistoquímica e zimográfica precoce, sendo sugeridas como fator prognóstico, detectando principalmente a capacidade de invasão das lesões. Já, no tratamento do câncer, as MMP são foco por serem alvo da indústria farmacêutica para a confecção de inibidores das MMP, os quais têm sido avaliados por diversos autores. Portanto, sabe-se da complexidade das características dessas enzimas e da necessidade de novos estudos, visando um futuro promissor das pesquisas.

\section{REFERÊNCIAS BIBLIOGRÁFICAS}

1. Instituto Nacional de Câncer; Ministério da Saúde. Estimativa 2005 [monografia na internet]. Rio de Janeiro: INCA; c 1996-2006. [citado em 2006 Mar 17]. Disponível em: http://www.inca.gov.br/estimativa/2005/ index.asp?link=conteudo_view.asp\&ID=1 .

2. Brasileiro Filho G. Distúrbios do crescimento e da diferenciação celular. In: Brasileiro Filho G. Bogliolo patologia geral. 3 th ed. Rio de Janeiro: Guanabara-Koogan; 2004. p.173-234.

3. Montenegro MR. Neoplasias. In: Montenegro MR, Franco M. Patologia. Processos gerais. $3^{a}$ ed. Rio de Janeiro: Atheneu; 1995. p.205-16.

4. Goljan EF. Neoplasias. In: Goljan EF. Resumo de patologia. São Paulo: Roca; 2002. p.97-105.

5. Kerkela E, Saarialho-Kere U. Matrix metalloproteinases in tumor progression: focus on basal and squamous cell skin cancer. Exp Dermatol. 2003 Apr;12(2):109-25.

6. Hong SD, Hong SP, Lee JI, Lim CY. Expression of matrix metalloproteinase-2 and -9 in oral squamous cell carcinomas with regard to the metastatic potential. Oral Oncol. 2000 Mar;36(2):207-13.

7. Ohtani H. Stromal reaction in cancer tissue: pathophysiologic significance of the expression of matrixdegrading enzymes in relation to matrix turnover and immune/inflammatory reactions. Pathol Int. 1998 Jan;48(1):1-9.

8. Thomas GT, Lewis MP, Speight PM. Matrix metalloproteinases and oral cancer. Oral Oncol. 1999 May;35(3):227-33.

9. Stenback F, Makinen MJ, Jussila T, Kauppila S, Risteli J, Talve L, et al. The extracellular matrix in skin tumor development-a morphological study. J Cutan Pathol. 1999 Aug;26(7):327-38.

10. Sternlicht MD, Werb Z. How matrix metalloproteinases regulate cell behavior. Annu Rev Cell Dev Biol. 2001;17:463-516.

11. Visse R, Nagase H. Matrix metalloproteinases and tissue inhibitors of metalloproteinases: structure, function, and biochemistry. Circ Res. 2003 May 2;92(8):827-39.
12. Li WP, Anderson CJ. Imaging matrix metalloproteinase expression in tumors. QJ Nucl Med. 2003 Sep;47(3):201-8.

13. Woessner JF Jr. Matrix metalloproteinases and their inhibitors in connective tissue remodeling. FASEB J. 1991 May;5(8):2145-54.

14. Woessner JF Jr. The matrix metalloproteinase family. In: Parks WC, Mecham RP. Matrix metalloproteinases. San Diego: Academic Press; 1998. p.1-14.

15. Hidalgo M, Eckhardt SG. Development of matrix metalloproteinase inhibitors in cancer therapy. J Natl Cancer Inst. 2001 Feb 7;93(3):178-93.

16. Birkedal-Hansen H. Proteolytic remodeling of extracellular matrix. Curr Opin Cell Biol. 1995 Oct;7(5):728-35.

17. Nabeshima K, Inoue T, Shimao Y, Sameshima T. Matrix metalloproteinases in tumor invasion: role for cell migration. Pathol Int. 2002 Apr;52(4):255-64.

18. Iki K, Tsutsumi M, Kido A, Sakitani H, Takahama M, Yoshimoto M, et al. Expression of matrix metalloproteinase 2 (MMP-2), membrane-type 1 MMP and tissue inhibitor of metalloproteinase 2 and activation of proMMP- 2 in pancreatic duct adenocarcinomas in hamsters treated with N-nitrosobis(2-oxopropyl)amine. Carcinogenesis. 1999 Jul;20(7):1323-9.

19. Fini ME, Cook JR, Mohan R, BrinckerhoffCE. Regulation of matrix metalloproteinase gene expression. In: Parks WC, Mecham RP. Matrix metalloproteinases. San Diego: Academic Press; 1998. p.299-356.

20. Rothenberg ML, Nelson AR, Hande KR. New drugs on the horizon: matrix metalloproteinase inhibitors. Stem Cells. 1999;17(4):237-40.

21. Duffy MJ, Maguire TM, Hill A, McDermott E, O'Higgins N. Metalloproteinases: role in breast carcinogenesis, invasion and metastasis. Breast Cancer Res. 2000;2(4):252-7.

22. Maskos K, Bode W. Structural basis of matrix metalloproteinases and tissue inhibitors of metalloproteinases. Mol Biotechnol. 2003 Nov;25(3):241-66.

23. Parks WC, Sudbeck BD, Doyle GR, Saarialho-Kere UK. Matrix metalloproteinases in tissue repair. In: Parks WC, Mecham RP. Matrix metalloproteinases. San Diego: Academic Press; 1998. p.263-98.

24. Wylie S, MacDonald IC, Varghese HJ, Schmidt EE, Morris VL, Groom AC, et al. The matrix metalloproteinase inhibitor batimastat inhibits angiogenesis in liver metastases of B16F1 melanoma cells. Clin Exp Metastasis. 1999 Mar;17(2):111-7.

25. Maekawa R, Maki H, Wada T, Yoshida H, NishidaNishimoto K, Okamoto H, et al. Anti-metastatic efficacy and safety of MMI-166, a selective matrix metalloproteinase inhibitor. Clin Exp Metastasis. 2000;18(1):61-6.

26. Maki H, Hojo K, Tanaka H, Sawada TY, Maekawa R, Yoshioka T. Augmented anti-metastatic efficacy of a selective 
matrix metalloproteinase inhibitor, MMI-166, in combination with CPT-11. Clin Exp Metastasis. 2002;19(6):519-26.

27. YuAE, Murphy AN, Stetler-Stevenson WG. 72-kDa Gelatinase (Gelatinase A): structure, activation, regulation and substrate specificity. In: Parks WC, Mecham RP. Matrix metalloproteinases. San Diego: Academic Press; 1998. p.85-114.

28. Fundyler O, Khanna M, Smoller BR. Metalloproteinase-2 expression correlates with aggressiveness of cutaneous squamous cell carcinomas. Mod Pathol. 2004 May;17(5):496-502.

29. Koyama H, Iwata H, Kuwabara Y, Iwase H, Kobayashi S, Fujii Y. Gelatinolytic activity of matrix metalloproteinase-2 and -9 in oesophageal carcinoma; a study using in situ zymography. Eur J Cancer. 2000 Oct;36(16):2164-70.

30. Lin SC, Lo SS, Liu CJ, Chung MY, Huang JW, Chang KW. Functional genotype in matrix metalloproteinases-2 promoter is a risk factor for oral carcinogenesis. J Oral Pathol Med. 2004 Aug;33(7):405-9.

31. Lin SC, Chung MY, Huang JW, Shieh TM, Liu CJ, Chang KW. Correlation between functional genotypes in the matrix metalloproteinases-1 promoter and risk of oral squamous cell carcinomas. J Oral Pathol Med. 2004 Jul;33(6):323-6.

32. Aglund K, Rauvala M, Puistola U, Angstrom T, Turpeenniemi-Hujanen T, Zackrisson B. et al. Gelatinases $A$ and B (MMP-2 and MMP-9) in endometrial cancerMMP-9 correlates to the grade and the stage. Gynecol Oncol. 2004 Sep;94(3):699-704.

33. Liu WW, Zeng ZY, Wu QL, Hou JH, Chen YY. Overexpression of MMP-2 in laryngeal squamous cell carcinoma: a potential indicator for poor prognosis. Otolaryngol Head Neck Surg. 2005 Mar;132(3):395-400.

34. Kawamata H, Kameyama S, Kawai K, Tanaka Y, Nan L, Barch DH et al. Marked acceleration of the metastatic phenotype of a rat bladder carcinoma cell line by the expression of human gelatinase A. Int J Cancer. 1995 Nov;63(4):568-75.

35. Bernhard EJ, Gruber SB, Muschel RJ. Direct evidence linking expression of matrix metalloproteinase $9(92-\mathrm{kDa}$ gelatinase/collagenase) to the metastatic phenotype in transformed rat embryo cells. Proc Natl Acad Sci U S A. 1994 May;91(10):4293-7.

36. Hua J, Muschel RJ. Inhibition of matrix metalloproteinase 9 expression by a ribozyme blocks metastasis in a rat sarcoma model system. Cancer Res. 1996 Nov;56(22):5279-84.

37. Ikebe T, Shinohara M, Takeuchi H, Beppu M, Kurahara S, Nakamura S, et al. Gelatinolytic activity of matrix metalloproteinase in tumor tissues correlates with the invasiveness of oral cancer. Clin Exp Metastasis. 1999 Jun;17(4):315-23.

38. Stetler-Stevenson WG, Aznavoorian S, Liotta A. Tumor cell interactions with the extracellular matrix during invasion and metastasis. Annu Rev Cell Biol. 1993; 9:541-73.

39. Santos O, McDermott CD, Daniels RG, Appelt K. Rodent pharmacokinetic and anti-tumor efficacy studies with a series of synthetic inhibitors of matrix metalloproteinases. Clin Exp Metastasis. 1997 Sep;15(5):499-508.

40. Kido A, Tsutsumi M, Iki K, Motoyama M, Takahama M, Tsujiuchi $\mathrm{T}$, et al. Inhibition of spontaneous rat osteosarcoma lung metastasis by 3S-[4-(N-hydroxyamino)2R-isobutylsuccinyl] amino-1-methoxy-3,4-dihydroc arbostyril, a novel matrix metalloproteinase inhibitor. Jpn J Cancer Res. 1999 Mar;90(3):333-41.

41. Nagase H, Woessner JF Jr. Matrix metalloproteinases. J Biol Chem. 1999 Jul 30;274(31):21491-4.

42. McCawley LJ, Matrisian LM. Matrix metalloproteinases: they're not just for matrix anymore! Curr Opin Cell Biol. 2001 Oct;13(5):534-40. 